Acta Crystallographica Section E

Structure Reports

Online

ISSN 1600-5368

\section{2-[4-tert-Butyl-5-(2-chlorobenzyl)-1,3- thiazol-2-yl]isoindoline-1,3-dione}

\section{Zhi-Gang Yao, ${ }^{\text {a Jun-Mei Peng, }}{ }^{\text {b }}$ Su-Fang Huo ${ }^{b}$ and Ai-Xi $\mathbf{H u}^{\mathrm{a} *}$}

${ }^{a}$ School of Chemistry and Chemical Engineering, South China University of Technology, Guangzhou 510640, People's Republic of China, and ${ }^{\mathbf{b}}$ College of Chemistry and Chemical Engineering, Hunan University, Changsha 410082, People's Republic of China

Correspondence e-mail: axhu0731@yahoo.com.cn

Received 18 October 2010; accepted 20 October 2010

Key indicators: single-crystal X-ray study; $T=173 \mathrm{~K}$; mean $\sigma(\mathrm{C}-\mathrm{C})=0.003 \AA$; $R$ factor $=0.036 ; w R$ factor $=0.122 ;$ data-to-parameter ratio $=15.1$.

In the title compound, $\mathrm{C}_{22} \mathrm{H}_{19} \mathrm{ClN}_{2} \mathrm{O}_{2} \mathrm{~S}$, the dihedral angle between the phenylene ring and the phthalimide ring system is $4.4(1)^{\circ}$. There is no hydrogen bonding or $\pi-\pi$ stacking in the crystal structure.

\section{Related literature}

For background to thiazole derivatives, see: Kazzouli et al. (2002); Holla et al. (2003); Hu et al. (2008), For background to phthalimide derivatives, see: Lima et al. (2002); Miyachi et al. (1997); Yachide et al. (2007).<smiles>CC(C)(C)c1nc(N2C(=O)c3ccccc3C2=O)sc1Cc1ccccc1Cl</smiles>

\section{Experimental}

Crystal data

$\mathrm{C}_{22} \mathrm{H}_{19} \mathrm{ClN}_{2} \mathrm{O}_{2} \mathrm{~S} \quad M_{r}=410.90$
Triclinic, $P \overline{1}$

$a=7.8357$ (4) $\AA$

$b=8.1587$ (4) $\AA$

$c=16.1487(8) \AA$

$\alpha=100.404$ (1)

$\beta=95.897$ (1)

$\gamma=96.490(1)^{\circ}$

$V=1000.85(9) \AA^{3}$

$Z=2$

Mo $K \alpha$ radiation

$\mu=0.32 \mathrm{~mm}^{-1}$

$T=173 \mathrm{~K}$

$0.46 \times 0.30 \times 0.28 \mathrm{~mm}$

\section{Data collection}

Bruker SMART 1000 CCD diffractometer

Absorption correction: multi-scan (SADABS; Sheldrick, 2004)

$T_{\min }=0.868, T_{\max }=0.917$

Refinement

$R\left[F^{2}>2 \sigma\left(F^{2}\right)\right]=0.036$

$w R\left(F^{2}\right)=0.122$

$S=1.15$

3857 reflections

7798 measured reflections 3857 independent reflections 3268 reflections with $I>2 \sigma(I)$ $R_{\text {int }}=0.018$

Data collection: SMART (Bruker, 2001); cell refinement: SAINTPlus (Bruker, 2003); data reduction: SAINT-Plus; program(s) used to solve structure: SHELXS97 (Sheldrick, 2008); program(s) used to refine structure: SHELXL97 (Sheldrick, 2008); molecular graphics: SHELXTL (Sheldrick, 2008); software used to prepare material for publication: SHELXTL.

Supplementary data and figures for this paper are available from the IUCr electronic archives (Reference: NG5049).

\section{References}

Bruker (2001). SMART. Bruker AXS Inc., Madison, Wisconsin, USA.

Bruker (2003). SAINT-Plus. Bruker AXS Inc., Madison, Wisconsin, USA.

Holla, B. S., Malini, K. V., Rao, B. S. N., Sarojini, B. K. \& Kumari, N. S. (2003). Eur. J. Med. Chem. 38, 313-318.

Hu, A. X., Cao, G., Ma, Y. Q., Zhang, J. Y. \& Ou, X. M. (2008). Chin. J. Struct. Chem. 27, 1235-1239.

Kazzouli, S. E., Berteina-Raboin, S., Mouaddibb, A. \& Guillaumeta, G. (2002). Tetrahedron Lett. 43, 3193-3196.

Lima, L. M., Castro, P., Machado, A. L., Fraga, C. A. M., Lugnier, C., Moraes, V. L. G. \& Barreiro, E. J. (2002). Bioorg. Med. Chem. 12, 3067-3073.

Miyachi, H., Ogasawara, A., Azuma, A. \& Hashimoto, Y. (1997). Bioorg. Med. Chem. 5, 2095-2102.

Sheldrick, G. M. (2004). SADABS. University of Göttingen, Germany.

Sheldrick, G. M. (2008). Acta Cryst. A64, 112-122.

Yachide, T. N., Aoyama, A., Makishima, M., Miyachi, H. \& Hashimoto, Y. (2007). Bioorg. Med. Chem Lett. 17, 3957-3961. 


\section{supporting information}

Acta Cryst. (2010). E66, o2940［https://doi.org/10.1107/S1600536810042601]

\section{2-[4-tert-Butyl-5-(2-chlorobenzyl)-1,3-thiazol-2-yl] isoindoline-1,3-dione}

\section{Zhi-Gang Yao, Jun-Mei Peng, Su-Fang Huo and Ai-Xi Hu}

\section{S1. Comment}

Compounds containing thiazole ring have a wide spectrum of biological activities, many of them are well known as antiviral, antifungal agents (Kazzouli et al., 2002; Holla et al., 2003; Hu et al., 2008). $N$-substituted phthalimide derivatives are very important in pharmaceutical intermediates and drugs (Miyachi et al., 1997). Herein we report the synthesis and the crystal structure of the phthalimide compounds which contain the thiazole ring.

The molecular structure of the title compound, $\mathrm{C}_{22} \mathrm{H}_{19} \mathrm{ClN}_{2} \mathrm{O}_{2} \mathrm{~S}$, is shown in Fig 1. There are no hydrogen bonding and $\pi-\pi$ stacking in the crystal structure. The van der Waals interactions maintain the structural cohesion.

\section{S2. Experimental}

A solution of $10 \mathrm{mmol} 5$-(2-chlorobenzyl)-4-tert-butylthiazol-2-amine and $10 \mathrm{mmol}$ ph thalic anhydride in $15 \mathrm{ml}$ acetic acid, then heated and refluxed for $23 \mathrm{~h}$. After finishing the reaction, cooled the solution, and the precipitate formed, filtered, recrystallized with ethanol to give the title compound. The crystals for X-ray structure determination were obtained by slow evaporation of an ethanol solution at room temperature.

\section{S3. Refinement}

The crystal system of the title compound was triclinic and all $\mathrm{H}$ atoms were refined using riding mode. The $\mathrm{C}-\mathrm{H}$ distances of phenyl and tert-butyl were $0.95 \AA$ and $0.98 \AA$, with $U_{\text {iso }}(H)=1.5 U_{\mathrm{eq}}\left(\mathrm{C}_{\mathrm{methyl}}\right)$.

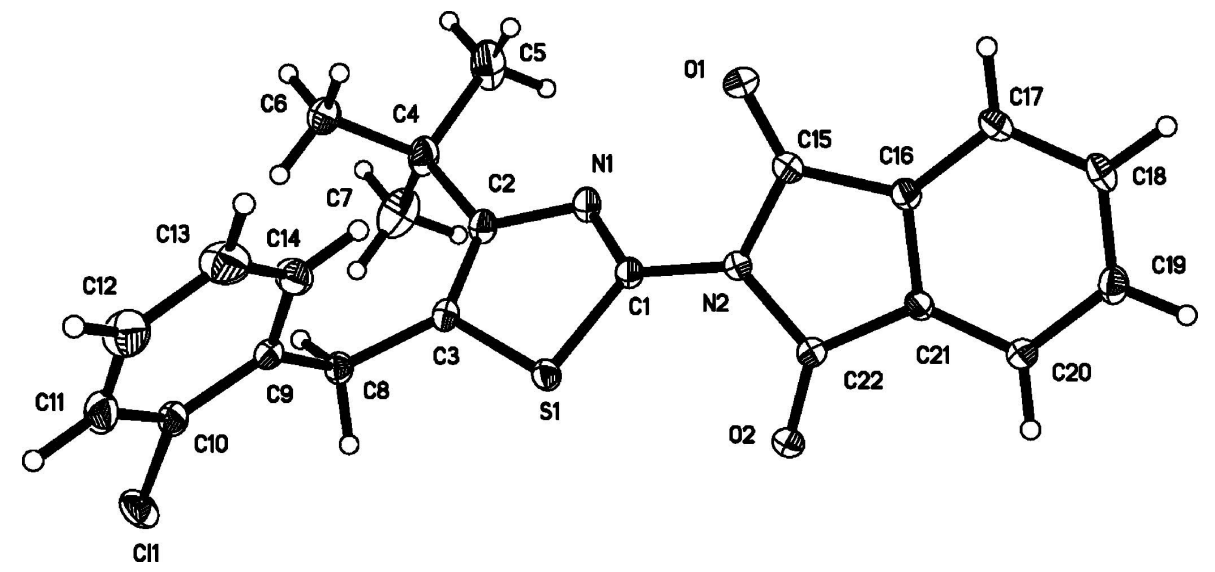

Figure 1

The molecular structure of the title compound, showing the atom-labelling scheme and $50 \%$ probability displacement ellipsoid (arbitrary spheres for $\mathrm{H}$ atoms). 


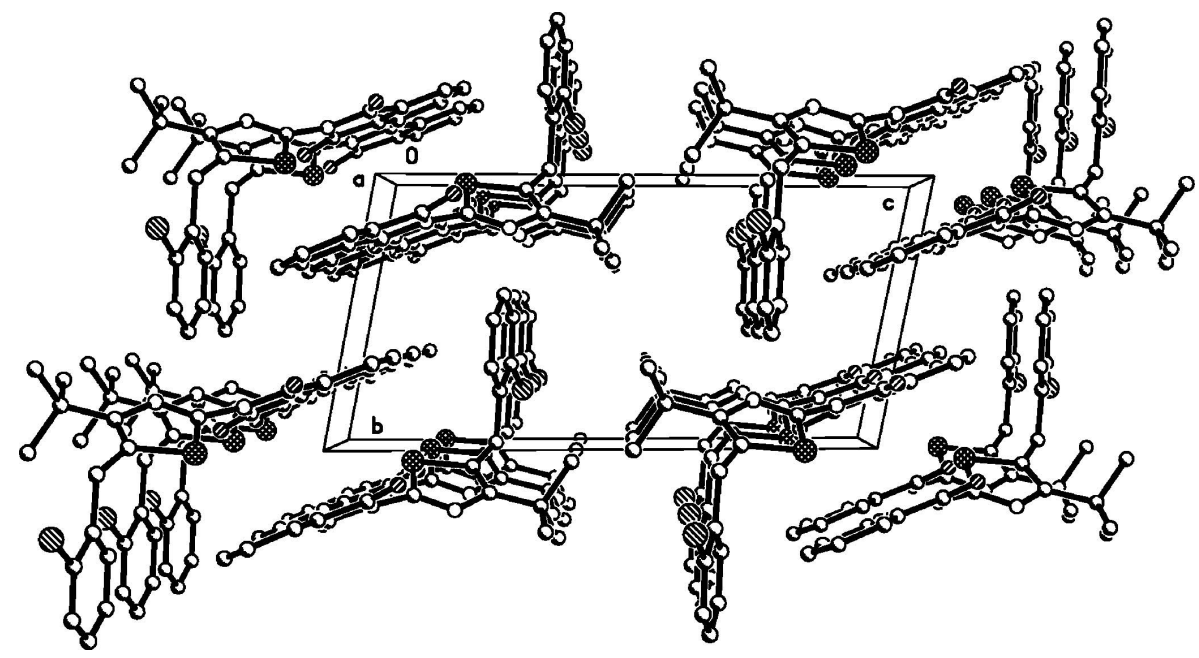

Figure 2

A packing diagram for the title compound.

\section{2-[4-tert-Butyl-5-(2-chlorobenzyl)-1,3-thiazol-2-yl]isoindoline-1,3-dione}

\section{Crystal data}

\section{$\mathrm{C}_{22} \mathrm{H}_{19} \mathrm{ClN}_{2} \mathrm{O}_{2} \mathrm{~S}$}

$M_{r}=410.90$

Triclinic, $P \overline{1}$

Hall symbol: -P 1

$a=7.8357(4) \AA$

$b=8.1587(4) \AA$

$c=16.1487(8) \AA$

$\alpha=100.404(1)^{\circ}$

$\beta=95.897(1)^{\circ}$

$\gamma=96.490(1)^{\circ}$

$V=1000.85(9) \AA^{3}$

\section{Data collection}

\section{Bruker SMART 1000 CCD}

diffractometer

Radiation source: fine-focus sealed tube

Graphite monochromator

$\omega$ scans

Absorption correction: multi-scan

(SADABS; Sheldrick, 2004)

$T_{\min }=0.868, T_{\max }=0.917$

\section{Refinement}

Refinement on $F^{2}$

Least-squares matrix: full

$R\left[F^{2}>2 \sigma\left(F^{2}\right)\right]=0.036$

$w R\left(F^{2}\right)=0.122$

$S=1.15$

3857 reflections

256 parameters

0 restraints
$Z=2$

$F(000)=428$

$D_{\mathrm{x}}=1.363 \mathrm{Mg} \mathrm{m}^{-3}$

Melting point: $425 \mathrm{~K}$

Mo $K \alpha$ radiation, $\lambda=0.71073 \AA$

Cell parameters from 5125 reflections

$\theta=2.6-27.0^{\circ}$

$\mu=0.32 \mathrm{~mm}^{-1}$

$T=173 \mathrm{~K}$

Block, colorless

$0.46 \times 0.30 \times 0.28 \mathrm{~mm}$

7798 measured reflections

3857 independent reflections

3268 reflections with $I>2 \sigma(I)$

$R_{\text {int }}=0.018$

$\theta_{\max }=26.0^{\circ}, \theta_{\min }=2.6^{\circ}$

$h=-9 \rightarrow 9$

$k=-10 \rightarrow 10$

$l=-19 \rightarrow 19$

Primary atom site location: structure-invariant direct methods

Secondary atom site location: difference Fourier map

Hydrogen site location: inferred from neighbouring sites

$\mathrm{H}$-atom parameters constrained 
$w=1 /\left[\sigma^{2}\left(F_{\mathrm{o}}^{2}\right)+(0.0649 P)^{2}+0.4002 P\right]$

where $P=\left(F_{\mathrm{o}}^{2}+2 F_{\mathrm{c}}^{2}\right) / 3$

$(\Delta / \sigma)_{\max }<0.001$

$$
\Delta \rho_{\max }=0.33 \mathrm{e} \AA^{-3}
$$

$\Delta \rho_{\min }=-0.31$ e $\AA^{-3}$

\section{Special details}

Experimental. The ${ }^{1} \mathrm{HNMR}\left(\mathrm{CDCl}_{3}, 400 \mathrm{MHz}\right)$ of the title compound were: $1.46\left(\mathrm{~s}, 9 \mathrm{H}, 3 \times \mathrm{CH}_{3}\right), 4.41\left(\mathrm{~s}, 2 \mathrm{H}, \mathrm{CH}_{2}\right)$, 7.18 7.39 (m, 4H, $\left.\mathrm{C}_{6} \mathrm{H}_{4}\right), 7.79 \sim 7.96\left(\mathrm{~m}, 4 \mathrm{H}, \mathrm{C}_{6} \mathrm{H}_{4}\right)$. And the yield was: 67.6\%. m.p.423 427 K.

Geometry. All e.s.d.'s (except the e.s.d. in the dihedral angle between two 1.s. planes) are estimated using the full covariance matrix. The cell e.s.d.'s are taken into account individually in the estimation of e.s.d.'s in distances, angles and torsion angles; correlations between e.s.d.'s in cell parameters are only used when they are defined by crystal symmetry. An approximate (isotropic) treatment of cell e.s.d.'s is used for estimating e.s.d.'s involving 1.s. planes.

Refinement. Refinement of $F^{2}$ against ALL reflections. The weighted $R$-factor $w R$ and goodness of fit $S$ are based on $F^{2}$, conventional $R$-factors $R$ are based on $F$, with $F$ set to zero for negative $F^{2}$. The threshold expression of $F^{2}>\sigma\left(F^{2}\right)$ is used only for calculating $R$-factors(gt) etc. and is not relevant to the choice of reflections for refinement. $R$-factors based on $F^{2}$ are statistically about twice as large as those based on $F$, and $R$ - factors based on ALL data will be even larger.

Fractional atomic coordinates and isotropic or equivalent isotropic displacement parameters $\left(\hat{A}^{2}\right)$

\begin{tabular}{lllll}
\hline & $x$ & $y$ & $z$ & $U_{\text {iso }} / U_{\text {eq }}$ \\
\hline C11 & $-0.47492(7)$ & $-0.21014(8)$ & $0.32706(3)$ & $0.04128(18)$ \\
S1 & $0.02664(6)$ & $0.03724(6)$ & $0.16354(3)$ & $0.02634(15)$ \\
C1 & $0.2305(2)$ & $0.1497(2)$ & $0.18229(12)$ & $0.0236(4)$ \\
C2 & $0.1795(3)$ & $0.1461(2)$ & $0.31377(12)$ & $0.0266(4)$ \\
C3 & $0.0266(2)$ & $0.0574(2)$ & $0.27220(12)$ & $0.0239(4)$ \\
C4 & $0.2332(3)$ & $0.1918(3)$ & $0.40993(13)$ & $0.0366(5)$ \\
C5 & $0.4226(4)$ & $0.2725(4)$ & $0.42868(16)$ & $0.0624(9)$ \\
H5A & 0.4953 & 0.1967 & 0.3993 & $0.094^{*}$ \\
H5B & 0.4592 & 0.2932 & 0.4900 & $0.094^{*}$ \\
H5C & 0.4347 & 0.3793 & 0.4088 & $0.094^{*}$ \\
C6 & $0.2175(3)$ & $0.0330(3)$ & $0.44847(14)$ & $0.0389(5)$ \\
H6A & 0.0978 & -0.0232 & 0.4360 & $0.058^{*}$ \\
H6B & 0.2495 & 0.0640 & 0.5101 & $0.058^{*}$ \\
H6C & 0.2950 & -0.0434 & 0.4240 & $0.058^{*}$ \\
C7 & $0.1182(4)$ & $0.3151(3)$ & $0.45113(15)$ & $0.0555(7)$ \\
H7A & 0.1307 & 0.4177 & 0.4275 & $0.083^{*}$ \\
H7B & 0.1534 & 0.3433 & 0.5126 & $0.083^{*}$ \\
H7C & -0.0029 & 0.2630 & 0.4396 & $0.083^{*}$ \\
C8 & $-0.1313(2)$ & $-0.0235(2)$ & $0.30286(12)$ & $0.0261(4)$ \\
H8A & -0.2359 & 0.0057 & 0.2720 & $0.031^{*}$ \\
H8B & -0.1298 & 0.0241 & 0.3639 & $0.031^{*}$ \\
C9 & $-0.1445(2)$ & $-0.2135(2)$ & $0.29113(12)$ & $0.0255(4)$ \\
C10 & $-0.2985(3)$ & $-0.3103(3)$ & $0.29935(12)$ & $0.0298(4)$ \\
C11 & $-0.3166(3)$ & $-0.4842(3)$ & $0.28567(15)$ & $0.0422(6)$ \\
H11 & -0.4235 & -0.5467 & 0.2909 & $0.051^{*}$ \\
C12 & $-0.1785(4)$ & $-0.5656(3)$ & $0.26456(18)$ & $0.0510(7)$ \\
H12 & -0.1901 & -0.6849 & 0.2547 & $0.061^{*}$ \\
C13 & $-0.0231(3)$ & $-0.4749(3)$ & $0.25766(18)$ & $0.0492(6)$ \\
H13 & 0.0730 & -0.5313 & 0.2441 & $0.059^{*}$ \\
C14 & $-0.0074(3)$ & $-0.3009(3)$ & $0.27052(15)$ & $0.0349(5)$ \\
& & & &
\end{tabular}




$\begin{array}{lllll}\mathrm{H} 14 & 0.1001 & -0.2396 & 0.2651 & 0.042^{*} \\ \mathrm{C} 15 & 0.4999(2) & 0.1549(2) & 0.11270(12) & 0.0248(4) \\ \mathrm{C} 16 & 0.5493(2) & 0.2158(2) & 0.03643(12) & 0.0245(4) \\ \mathrm{C} 17 & 0.7093(3) & 0.2341(3) & 0.00803(14) & 0.0306(4) \\ \mathrm{H} 17 & 0.8079 & 0.2015 & 0.0369 & 0.037^{*} \\ \mathrm{C} 18 & 0.7203(3) & 0.3020(3) & -0.06437(13) & 0.0319(5) \\ \mathrm{H} 18 & 0.8292 & 0.3190 & -0.0847 & 0.038^{*} \\ \mathrm{C} 19 & 0.5758(3) & 0.3453(3) & -0.10749(13) & 0.0307(4) \\ \mathrm{H} 19 & 0.5873 & 0.3896 & -0.1574 & 0.037^{*} \\ \mathrm{C} 20 & 0.4131(2) & 0.3257(3) & -0.07953(12) & 0.0270(4) \\ \mathrm{H} 20 & 0.3135 & 0.3546 & -0.1094 & 0.032^{*} \\ \mathrm{C} 21 & 0.4048(2) & 0.2618(2) & -0.00597(11) & 0.0228(4) \\ \mathrm{C} 22 & 0.2572(2) & 0.2381(2) & 0.04291(12) & 0.0249(4) \\ \mathrm{N} 1 & 0.2936(2) & 0.2002(2) & 0.26111(10) & 0.0276(4) \\ \mathrm{N} 2 & 0.3239(2) & 0.1807(2) & 0.11564(10) & 0.0244(3) \\ \text { O1 } & 0.58277(18) & 0.09357(19) & 0.16259(9) & 0.0334(3) \\ \text { O2 } & 0.10930(18) & 0.2615(2) & 0.02800(9) & 0.0374(4)\end{array}$

Atomic displacement parameters $\left(\AA^{2}\right)$

\begin{tabular}{lllllll}
\hline & $U^{11}$ & $U^{22}$ & $U^{33}$ & $U^{12}$ & $U^{13}$ & $U^{23}$ \\
\hline C11 & $0.0275(3)$ & $0.0591(4)$ & $0.0341(3)$ & $-0.0019(2)$ & $0.0104(2)$ & $0.0021(2)$ \\
S1 & $0.0230(3)$ & $0.0335(3)$ & $0.0218(3)$ & $-0.00074(19)$ & $0.00394(18)$ & $0.00557(19)$ \\
C1 & $0.0242(9)$ & $0.0245(9)$ & $0.0229(9)$ & $0.0027(7)$ & $0.0047(7)$ & $0.0062(7)$ \\
C2 & $0.0319(10)$ & $0.0250(10)$ & $0.0225(10)$ & $0.0002(8)$ & $0.0058(8)$ & $0.0043(7)$ \\
C3 & $0.0285(10)$ & $0.0230(9)$ & $0.0224(9)$ & $0.0048(7)$ & $0.0070(8)$ & $0.0071(7)$ \\
C4 & $0.0447(13)$ & $0.0408(12)$ & $0.0211(10)$ & $-0.0054(10)$ & $0.0049(9)$ & $0.0044(9)$ \\
C5 & $0.0642(18)$ & $0.083(2)$ & $0.0264(12)$ & $-0.0353(15)$ & $-0.0045(12)$ & $0.0075(12)$ \\
C6 & $0.0412(13)$ & $0.0510(14)$ & $0.0266(11)$ & $0.0052(10)$ & $0.0068(9)$ & $0.0125(10)$ \\
C7 & $0.092(2)$ & $0.0431(14)$ & $0.0298(12)$ & $0.0145(14)$ & $0.0097(13)$ & $-0.0021(10)$ \\
C8 & $0.0262(10)$ & $0.0280(10)$ & $0.0266(10)$ & $0.0047(8)$ & $0.0073(8)$ & $0.0086(8)$ \\
C9 & $0.0261(10)$ & $0.0284(10)$ & $0.0225(9)$ & $0.0018(8)$ & $0.0003(7)$ & $0.0093(8)$ \\
C10 & $0.0302(10)$ & $0.0370(11)$ & $0.0217(9)$ & $-0.0027(8)$ & $0.0026(8)$ & $0.0090(8)$ \\
C11 & $0.0456(13)$ & $0.0381(13)$ & $0.0398(13)$ & $-0.0132(10)$ & $-0.0033(10)$ & $0.0154(10)$ \\
C12 & $0.0573(16)$ & $0.0255(11)$ & $0.0673(17)$ & $-0.0004(11)$ & $-0.0070(13)$ & $0.0134(11)$ \\
C13 & $0.0443(14)$ & $0.0326(12)$ & $0.0706(18)$ & $0.0120(10)$ & $-0.0009(12)$ & $0.0105(12)$ \\
C14 & $0.0297(11)$ & $0.0294(11)$ & $0.0465(13)$ & $0.0037(8)$ & $0.0038(9)$ & $0.0105(9)$ \\
C15 & $0.0214(9)$ & $0.0261(10)$ & $0.0266(10)$ & $0.0022(7)$ & $0.0031(7)$ & $0.0050(8)$ \\
C16 & $0.0234(9)$ & $0.0258(10)$ & $0.0242(9)$ & $0.0023(7)$ & $0.0047(7)$ & $0.0044(8)$ \\
C17 & $0.0198(9)$ & $0.0369(11)$ & $0.0366(11)$ & $0.0055(8)$ & $0.0064(8)$ & $0.0086(9)$ \\
C18 & $0.0247(10)$ & $0.0372(11)$ & $0.0350(11)$ & $0.0021(8)$ & $0.0135(9)$ & $0.0060(9)$ \\
C19 & $0.0345(11)$ & $0.0343(11)$ & $0.0227(10)$ & $-0.0003(8)$ & $0.0078(8)$ & $0.0045(8)$ \\
C20 & $0.0244(10)$ & $0.0340(11)$ & $0.0227(9)$ & $0.0033(8)$ & $0.0019(8)$ & $0.0062(8)$ \\
C21 & $0.0197(9)$ & $0.0260(10)$ & $0.0213(9)$ & $0.0006(7)$ & $0.0036(7)$ & $0.0023(7)$ \\
C22 & $0.0232(10)$ & $0.0290(10)$ & $0.0227(9)$ & $0.0022(7)$ & $0.0016(7)$ & $0.0068(8)$ \\
N1 & $0.0315(9)$ & $0.0268(8)$ & $0.0237(8)$ & $-0.0020(7)$ & $0.0060(7)$ & $0.0052(7)$ \\
N2 & $0.0205(8)$ & $0.0309(9)$ & $0.0236(8)$ & $0.0034(6)$ & $0.0039(6)$ & $0.0093(6)$ \\
O1 & $0.0284(7)$ & $0.0411(8)$ & $0.0343(8)$ & $0.0081(6)$ & $0.0003(6)$ & $0.0166(7)$
\end{tabular}




$\begin{array}{lllllll}\mathrm{O} 2 & 0.0208(7) & 0.0607(10) & 0.0362(8) & 0.0081(7) & 0.0049(6) & 0.0216(7)\end{array}$

Geometric parameters $(\AA, \stackrel{o}{)}$

\begin{tabular}{|c|c|c|c|}
\hline $\mathrm{C} 11-\mathrm{C} 10$ & $1.741(2)$ & $\mathrm{C} 9-\mathrm{C} 10$ & $1.397(3)$ \\
\hline $\mathrm{S} 1-\mathrm{C} 1$ & $1.7188(19)$ & $\mathrm{C} 10-\mathrm{C} 11$ & $1.385(3)$ \\
\hline $\mathrm{S} 1-\mathrm{C} 3$ & $1.7327(19)$ & $\mathrm{C} 11-\mathrm{C} 12$ & $1.373(4)$ \\
\hline $\mathrm{C} 1-\mathrm{N} 1$ & $1.292(2)$ & C11-H11 & 0.9500 \\
\hline $\mathrm{C} 1-\mathrm{N} 2$ & $1.406(2)$ & $\mathrm{C} 12-\mathrm{C} 13$ & $1.377(4)$ \\
\hline $\mathrm{C} 2-\mathrm{C} 3$ & $1.369(3)$ & $\mathrm{C} 12-\mathrm{H} 12$ & 0.9500 \\
\hline $\mathrm{C} 2-\mathrm{N} 1$ & $1.385(2)$ & $\mathrm{C} 13-\mathrm{C} 14$ & $1.386(3)$ \\
\hline $\mathrm{C} 2-\mathrm{C} 4$ & $1.532(3)$ & C13-H13 & 0.9500 \\
\hline $\mathrm{C} 3-\mathrm{C} 8$ & $1.507(3)$ & $\mathrm{C} 14-\mathrm{H} 14$ & 0.9500 \\
\hline $\mathrm{C} 4-\mathrm{C} 5$ & $1.531(3)$ & $\mathrm{C} 15-\mathrm{O} 1$ & $1.197(2)$ \\
\hline $\mathrm{C} 4-\mathrm{C} 6$ & $1.534(3)$ & $\mathrm{C} 15-\mathrm{N} 2$ & $1.422(2)$ \\
\hline $\mathrm{C} 4-\mathrm{C} 7$ & $1.534(3)$ & $\mathrm{C} 15-\mathrm{C} 16$ & $1.480(3)$ \\
\hline $\mathrm{C} 5-\mathrm{H} 5 \mathrm{~A}$ & 0.9800 & $\mathrm{C} 16-\mathrm{C} 17$ & $1.380(3)$ \\
\hline C5-H5B & 0.9800 & $\mathrm{C} 16-\mathrm{C} 21$ & $1.386(3)$ \\
\hline $\mathrm{C} 5-\mathrm{H} 5 \mathrm{C}$ & 0.9800 & $\mathrm{C} 17-\mathrm{C} 18$ & $1.388(3)$ \\
\hline C6-H6A & 0.9800 & C17-H17 & 0.9500 \\
\hline $\mathrm{C} 6-\mathrm{H} 6 \mathrm{~B}$ & 0.9800 & $\mathrm{C} 18-\mathrm{C} 19$ & $1.382(3)$ \\
\hline C6- $\mathrm{H} 6 \mathrm{C}$ & 0.9800 & C18-H18 & 0.9500 \\
\hline C7-H7A & 0.9800 & $\mathrm{C} 19-\mathrm{C} 20$ & $1.398(3)$ \\
\hline C7-H7B & 0.9800 & C19-H19 & 0.9500 \\
\hline $\mathrm{C} 7-\mathrm{H} 7 \mathrm{C}$ & 0.9800 & $\mathrm{C} 20-\mathrm{C} 21$ & $1.385(3)$ \\
\hline $\mathrm{C} 8-\mathrm{C} 9$ & $1.518(3)$ & $\mathrm{C} 20-\mathrm{H} 20$ & 0.9500 \\
\hline $\mathrm{C} 8-\mathrm{H} 8 \mathrm{~A}$ & 0.9900 & $\mathrm{C} 21-\mathrm{C} 22$ & $1.480(3)$ \\
\hline $\mathrm{C} 8-\mathrm{H} 8 \mathrm{~B}$ & 0.9900 & $\mathrm{C} 22-\mathrm{O} 2$ & $1.203(2)$ \\
\hline C9- $-\mathrm{C} 14$ & $1.391(3)$ & $\mathrm{C} 22-\mathrm{N} 2$ & $1.414(2)$ \\
\hline $\mathrm{C} 1-\mathrm{S} 1-\mathrm{C} 3$ & $88.77(9)$ & $\mathrm{C} 11-\mathrm{C} 10-\mathrm{C} 9$ & $122.3(2)$ \\
\hline $\mathrm{N} 1-\mathrm{C} 1-\mathrm{N} 2$ & $122.33(17)$ & $\mathrm{C} 11-\mathrm{C} 10-\mathrm{Cl1}$ & $118.45(17)$ \\
\hline $\mathrm{N} 1-\mathrm{C} 1-\mathrm{S} 1$ & $115.89(14)$ & $\mathrm{C} 9-\mathrm{C} 10-\mathrm{Cl}$ & $119.27(16)$ \\
\hline $\mathrm{N} 2-\mathrm{C} 1-\mathrm{S} 1$ & $121.76(14)$ & $\mathrm{C} 12-\mathrm{C} 11-\mathrm{C} 10$ & $119.4(2)$ \\
\hline $\mathrm{C} 3-\mathrm{C} 2-\mathrm{N} 1$ & $114.54(17)$ & $\mathrm{C} 12-\mathrm{C} 11-\mathrm{H} 11$ & 120.3 \\
\hline $\mathrm{C} 3-\mathrm{C} 2-\mathrm{C} 4$ & $127.00(18)$ & $\mathrm{C} 10-\mathrm{C} 11-\mathrm{H} 11$ & 120.3 \\
\hline $\mathrm{N} 1-\mathrm{C} 2-\mathrm{C} 4$ & $118.45(17)$ & $\mathrm{C} 11-\mathrm{C} 12-\mathrm{C} 13$ & $120.3(2)$ \\
\hline $\mathrm{C} 2-\mathrm{C} 3-\mathrm{C} 8$ & $132.65(17)$ & $\mathrm{C} 11-\mathrm{C} 12-\mathrm{H} 12$ & 119.9 \\
\hline $\mathrm{C} 2-\mathrm{C} 3-\mathrm{S} 1$ & $109.93(14)$ & $\mathrm{C} 13-\mathrm{C} 12-\mathrm{H} 12$ & 119.9 \\
\hline $\mathrm{C} 8-\mathrm{C} 3-\mathrm{S} 1$ & $117.41(14)$ & $\mathrm{C} 12-\mathrm{C} 13-\mathrm{C} 14$ & $119.8(2)$ \\
\hline $\mathrm{C} 5-\mathrm{C} 4-\mathrm{C} 2$ & $109.70(17)$ & $\mathrm{C} 12-\mathrm{C} 13-\mathrm{H} 13$ & 120.1 \\
\hline $\mathrm{C} 5-\mathrm{C} 4-\mathrm{C} 6$ & $107.7(2)$ & $\mathrm{C} 14-\mathrm{C} 13-\mathrm{H} 13$ & 120.1 \\
\hline $\mathrm{C} 2-\mathrm{C} 4-\mathrm{C} 6$ & $110.24(18)$ & $\mathrm{C} 13-\mathrm{C} 14-\mathrm{C} 9$ & $121.9(2)$ \\
\hline $\mathrm{C} 5-\mathrm{C} 4-\mathrm{C} 7$ & $109.5(2)$ & $\mathrm{C} 13-\mathrm{C} 14-\mathrm{H} 14$ & 119.1 \\
\hline $\mathrm{C} 2-\mathrm{C} 4-\mathrm{C} 7$ & 110.09 (19) & $\mathrm{C} 9-\mathrm{C} 14-\mathrm{H} 14$ & 119.1 \\
\hline $\mathrm{C} 6-\mathrm{C} 4-\mathrm{C} 7$ & $109.56(19)$ & $\mathrm{O} 1-\mathrm{C} 15-\mathrm{N} 2$ & $124.78(18)$ \\
\hline $\mathrm{C} 4-\mathrm{C} 5-\mathrm{H} 5 \mathrm{~A}$ & 109.5 & $\mathrm{O} 1-\mathrm{C} 15-\mathrm{C} 16$ & $129.95(18)$ \\
\hline $\mathrm{C} 4-\mathrm{C} 5-\mathrm{H} 5 \mathrm{~B}$ & 109.5 & $\mathrm{~N} 2-\mathrm{C} 15-\mathrm{C} 16$ & $105.26(15)$ \\
\hline
\end{tabular}




\begin{tabular}{|c|c|c|c|}
\hline $\mathrm{H} 5 \mathrm{~A}-\mathrm{C} 5-\mathrm{H} 5 \mathrm{~B}$ & 109.5 & $\mathrm{C} 17-\mathrm{C} 16-\mathrm{C} 21$ & $121.50(18)$ \\
\hline $\mathrm{C} 4-\mathrm{C} 5-\mathrm{H} 5 \mathrm{C}$ & 109.5 & $\mathrm{C} 17-\mathrm{C} 16-\mathrm{C} 15$ & $129.50(18)$ \\
\hline $\mathrm{H} 5 \mathrm{~A}-\mathrm{C} 5-\mathrm{H} 5 \mathrm{C}$ & 109.5 & $\mathrm{C} 21-\mathrm{C} 16-\mathrm{C} 15$ & $108.97(16)$ \\
\hline $\mathrm{H} 5 \mathrm{~B}-\mathrm{C} 5-\mathrm{H} 5 \mathrm{C}$ & 109.5 & $\mathrm{C} 16-\mathrm{C} 17-\mathrm{C} 18$ & $117.32(18)$ \\
\hline $\mathrm{C} 4-\mathrm{C} 6-\mathrm{H} 6 \mathrm{~A}$ & 109.5 & $\mathrm{C} 16-\mathrm{C} 17-\mathrm{H} 17$ & 121.3 \\
\hline $\mathrm{C} 4-\mathrm{C} 6-\mathrm{H} 6 \mathrm{~B}$ & 109.5 & $\mathrm{C} 18-\mathrm{C} 17-\mathrm{H} 17$ & 121.3 \\
\hline $\mathrm{H} 6 \mathrm{~A}-\mathrm{C} 6-\mathrm{H} 6 \mathrm{~B}$ & 109.5 & $\mathrm{C} 19-\mathrm{C} 18-\mathrm{C} 17$ & $121.24(18)$ \\
\hline $\mathrm{C} 4-\mathrm{C} 6-\mathrm{H} 6 \mathrm{C}$ & 109.5 & $\mathrm{C} 19-\mathrm{C} 18-\mathrm{H} 18$ & 119.4 \\
\hline $\mathrm{H} 6 \mathrm{~A}-\mathrm{C} 6-\mathrm{H} 6 \mathrm{C}$ & 109.5 & $\mathrm{C} 17-\mathrm{C} 18-\mathrm{H} 18$ & 119.4 \\
\hline $\mathrm{H} 6 \mathrm{~B}-\mathrm{C} 6-\mathrm{H} 6 \mathrm{C}$ & 109.5 & $\mathrm{C} 18-\mathrm{C} 19-\mathrm{C} 20$ & $121.71(19)$ \\
\hline $\mathrm{C} 4-\mathrm{C} 7-\mathrm{H} 7 \mathrm{~A}$ & 109.5 & $\mathrm{C} 18-\mathrm{C} 19-\mathrm{H} 19$ & 119.1 \\
\hline $\mathrm{C} 4-\mathrm{C} 7-\mathrm{H} 7 \mathrm{~B}$ & 109.5 & $\mathrm{C} 20-\mathrm{C} 19-\mathrm{H} 19$ & 119.1 \\
\hline $\mathrm{H} 7 \mathrm{~A}-\mathrm{C} 7-\mathrm{H} 7 \mathrm{~B}$ & 109.5 & $\mathrm{C} 21-\mathrm{C} 20-\mathrm{C} 19$ & $116.44(18)$ \\
\hline $\mathrm{C} 4-\mathrm{C} 7-\mathrm{H} 7 \mathrm{C}$ & 109.5 & $\mathrm{C} 21-\mathrm{C} 20-\mathrm{H} 20$ & 121.8 \\
\hline $\mathrm{H} 7 \mathrm{~A}-\mathrm{C} 7-\mathrm{H} 7 \mathrm{C}$ & 109.5 & $\mathrm{C} 19-\mathrm{C} 20-\mathrm{H} 20$ & 121.8 \\
\hline $\mathrm{H} 7 \mathrm{~B}-\mathrm{C} 7-\mathrm{H} 7 \mathrm{C}$ & 109.5 & $\mathrm{C} 20-\mathrm{C} 21-\mathrm{C} 16$ & $121.75(17)$ \\
\hline $\mathrm{C} 3-\mathrm{C} 8-\mathrm{C} 9$ & $113.97(16)$ & $\mathrm{C} 20-\mathrm{C} 21-\mathrm{C} 22$ & $129.59(17)$ \\
\hline $\mathrm{C} 3-\mathrm{C} 8-\mathrm{H} 8 \mathrm{~A}$ & 108.8 & $\mathrm{C} 16-\mathrm{C} 21-\mathrm{C} 22$ & $108.59(16)$ \\
\hline $\mathrm{C} 9-\mathrm{C} 8-\mathrm{H} 8 \mathrm{~A}$ & 108.8 & $\mathrm{O} 2-\mathrm{C} 22-\mathrm{N} 2$ & $124.31(18)$ \\
\hline $\mathrm{C} 3-\mathrm{C} 8-\mathrm{H} 8 \mathrm{~B}$ & 108.8 & $\mathrm{O} 2-\mathrm{C} 22-\mathrm{C} 21$ & $129.93(18)$ \\
\hline $\mathrm{C} 9-\mathrm{C} 8-\mathrm{H} 8 \mathrm{~B}$ & 108.8 & $\mathrm{~N} 2-\mathrm{C} 22-\mathrm{C} 21$ & $105.75(15)$ \\
\hline $\mathrm{H} 8 \mathrm{~A}-\mathrm{C} 8-\mathrm{H} 8 \mathrm{~B}$ & 107.7 & $\mathrm{C} 1-\mathrm{N} 1-\mathrm{C} 2$ & $110.85(16)$ \\
\hline $\mathrm{C} 14-\mathrm{C} 9-\mathrm{C} 10$ & 116.45 (19) & $\mathrm{C} 1-\mathrm{N} 2-\mathrm{C} 22$ & $125.37(16)$ \\
\hline $\mathrm{C} 14-\mathrm{C} 9-\mathrm{C} 8$ & $122.59(17)$ & $\mathrm{C} 1-\mathrm{N} 2-\mathrm{C} 15$ & $123.47(15)$ \\
\hline $\mathrm{C} 10-\mathrm{C} 9-\mathrm{C} 8$ & $120.96(18)$ & $\mathrm{C} 22-\mathrm{N} 2-\mathrm{C} 15$ & $111.15(15)$ \\
\hline $\mathrm{C} 3-\mathrm{S} 1-\mathrm{C} 1-\mathrm{N} 1$ & $-0.76(16)$ & $\mathrm{N} 2-\mathrm{C} 15-\mathrm{C} 16-\mathrm{C} 21$ & $-4.5(2)$ \\
\hline $\mathrm{C} 3-\mathrm{S} 1-\mathrm{C} 1-\mathrm{N} 2$ & $177.63(16)$ & $\mathrm{C} 21-\mathrm{C} 16-\mathrm{C} 17-\mathrm{C} 18$ & $0.6(3)$ \\
\hline $\mathrm{N} 1-\mathrm{C} 2-\mathrm{C} 3-\mathrm{C} 8$ & $-179.96(18)$ & $\mathrm{C} 15-\mathrm{C} 16-\mathrm{C} 17-\mathrm{C} 18$ & $-177.14(19)$ \\
\hline $\mathrm{C} 4-\mathrm{C} 2-\mathrm{C} 3-\mathrm{C} 8$ & $-0.9(4)$ & $\mathrm{C} 16-\mathrm{C} 17-\mathrm{C} 18-\mathrm{C} 19$ & $-1.7(3)$ \\
\hline $\mathrm{N} 1-\mathrm{C} 2-\mathrm{C} 3-\mathrm{S} 1$ & $1.1(2)$ & $\mathrm{C} 17-\mathrm{C} 18-\mathrm{C} 19-\mathrm{C} 20$ & $1.1(3)$ \\
\hline $\mathrm{C} 4-\mathrm{C} 2-\mathrm{C} 3-\mathrm{S} 1$ & $-179.79(17)$ & $\mathrm{C} 18-\mathrm{C} 19-\mathrm{C} 20-\mathrm{C} 21$ & $0.5(3)$ \\
\hline $\mathrm{C} 1-\mathrm{S} 1-\mathrm{C} 3-\mathrm{C} 2$ & $-0.25(15)$ & $\mathrm{C} 19-\mathrm{C} 20-\mathrm{C} 21-\mathrm{C} 16$ & $-1.6(3)$ \\
\hline $\mathrm{C} 1-\mathrm{S} 1-\mathrm{C} 3-\mathrm{C} 8$ & $-179.33(15)$ & $\mathrm{C} 19-\mathrm{C} 20-\mathrm{C} 21-\mathrm{C} 22$ & $175.01(18)$ \\
\hline $\mathrm{C} 3-\mathrm{C} 2-\mathrm{C} 4-\mathrm{C} 5$ & $173.1(2)$ & $\mathrm{C} 17-\mathrm{C} 16-\mathrm{C} 21-\mathrm{C} 20$ & $1.1(3)$ \\
\hline $\mathrm{N} 1-\mathrm{C} 2-\mathrm{C} 4-\mathrm{C} 5$ & $-7.9(3)$ & $\mathrm{C} 15-\mathrm{C} 16-\mathrm{C} 21-\mathrm{C} 20$ & $179.23(17)$ \\
\hline $\mathrm{C} 3-\mathrm{C} 2-\mathrm{C} 4-\mathrm{C} 6$ & $54.6(3)$ & $\mathrm{C} 17-\mathrm{C} 16-\mathrm{C} 21-\mathrm{C} 22$ & $-176.19(18)$ \\
\hline $\mathrm{N} 1-\mathrm{C} 2-\mathrm{C} 4-\mathrm{C} 6$ & $-126.4(2)$ & $\mathrm{C} 15-\mathrm{C} 16-\mathrm{C} 21-\mathrm{C} 22$ & $2.0(2)$ \\
\hline $\mathrm{C} 3-\mathrm{C} 2-\mathrm{C} 4-\mathrm{C} 7$ & $-66.4(3)$ & $\mathrm{C} 20-\mathrm{C} 21-\mathrm{C} 22-\mathrm{O} 2$ & $3.8(4)$ \\
\hline $\mathrm{N} 1-\mathrm{C} 2-\mathrm{C} 4-\mathrm{C} 7$ & $112.6(2)$ & $\mathrm{C} 16-\mathrm{C} 21-\mathrm{C} 22-\mathrm{O} 2$ & $-179.2(2)$ \\
\hline $\mathrm{C} 2-\mathrm{C} 3-\mathrm{C} 8-\mathrm{C} 9$ & $-104.1(2)$ & $\mathrm{C} 20-\mathrm{C} 21-\mathrm{C} 22-\mathrm{N} 2$ & $-175.65(19)$ \\
\hline $\mathrm{S} 1-\mathrm{C} 3-\mathrm{C} 8-\mathrm{C} 9$ & $74.74(19)$ & $\mathrm{C} 16-\mathrm{C} 21-\mathrm{C} 22-\mathrm{N} 2$ & $1.3(2)$ \\
\hline $\mathrm{C} 3-\mathrm{C} 8-\mathrm{C} 9-\mathrm{C} 14$ & $12.5(3)$ & $\mathrm{N} 2-\mathrm{C} 1-\mathrm{N} 1-\mathrm{C} 2$ & $-176.86(17)$ \\
\hline $\mathrm{C} 3-\mathrm{C} 8-\mathrm{C} 9-\mathrm{C} 10$ & $-166.43(18)$ & $\mathrm{S} 1-\mathrm{C} 1-\mathrm{N} 1-\mathrm{C} 2$ & $1.5(2)$ \\
\hline $\mathrm{C} 14-\mathrm{C} 9-\mathrm{C} 10-\mathrm{C} 11$ & $-1.7(3)$ & $\mathrm{C} 3-\mathrm{C} 2-\mathrm{N} 1-\mathrm{C} 1$ & $-1.7(2)$ \\
\hline $\mathrm{C} 8-\mathrm{C} 9-\mathrm{C} 10-\mathrm{C} 11$ & $177.34(19)$ & $\mathrm{C} 4-\mathrm{C} 2-\mathrm{N} 1-\mathrm{C} 1$ & $179.14(18)$ \\
\hline $\mathrm{C} 14-\mathrm{C} 9-\mathrm{C} 10-\mathrm{Cl1}$ & $178.77(15)$ & $\mathrm{N} 1-\mathrm{C} 1-\mathrm{N} 2-\mathrm{C} 22$ & $-134.9(2)$ \\
\hline $\mathrm{C} 8-\mathrm{C} 9-\mathrm{C} 10-\mathrm{Cl1}$ & $-2.2(3)$ & $\mathrm{S} 1-\mathrm{C} 1-\mathrm{N} 2-\mathrm{C} 22$ & $46.8(2)$ \\
\hline
\end{tabular}




$\begin{array}{llll}\mathrm{C} 9-\mathrm{C} 10-\mathrm{C} 11-\mathrm{C} 12 & 1.0(3) & \mathrm{N} 1-\mathrm{C} 1-\mathrm{N} 2-\mathrm{C} 15 & 46.1(3) \\ \mathrm{C} 11-\mathrm{C} 10-\mathrm{C} 11-\mathrm{C} 12 & -179.38(19) & \mathrm{S} 1-\mathrm{C} 1-\mathrm{N} 2-\mathrm{C} 15 & -132.20(17) \\ \mathrm{C} 10-\mathrm{C} 11-\mathrm{C} 12-\mathrm{C} 13 & 0.5(4) & \mathrm{O} 2-\mathrm{C} 22-\mathrm{N} 2-\mathrm{C} 1 & -2.9(3) \\ \mathrm{C} 11-\mathrm{C} 12-\mathrm{C} 13-\mathrm{C} 14 & -1.2(4) & \mathrm{C} 21-\mathrm{C} 22-\mathrm{N} 2-\mathrm{C} 1 & 176.62(16) \\ \mathrm{C} 12-\mathrm{C} 13-\mathrm{C} 14-\mathrm{C} 9 & 0.6(4) & \mathrm{O} 2-\mathrm{C} 22-\mathrm{N} 2-\mathrm{C} 15 & -4.3(2) \\ \mathrm{C} 10-\mathrm{C} 9-\mathrm{C} 14-\mathrm{C} 13 & 0.8(3) & \mathrm{C} 21-\mathrm{C} 22-\mathrm{N} 2-\mathrm{C} 15 & 5.6(3) \\ \mathrm{C} 8-\mathrm{C} 9-\mathrm{C} 14-\mathrm{C} 13 & -178.1(2) & \mathrm{O} 1-\mathrm{C} 15-\mathrm{N} 2-\mathrm{C} 1 & -175.46(16) \\ \mathrm{O} 1-\mathrm{C} 15-\mathrm{C} 16-\mathrm{C} 17 & -7.7(4) & \mathrm{C} 16-\mathrm{C} 15-\mathrm{N} 2-\mathrm{C} 1 & -173.47(19) \\ \mathrm{N} 2-\mathrm{C} 15-\mathrm{C} 16-\mathrm{C} 17 & 173.5(2) & \mathrm{O} 1-\mathrm{C} 15-\mathrm{N} 2-\mathrm{C} 22 & 5.4(2) \\ \mathrm{O} 1-\mathrm{C} 15-\mathrm{C} 16-\mathrm{C} 21 & 174.3(2) & \mathrm{C} 16-\mathrm{C} 15-\mathrm{N} 2-\mathrm{C} 22 & \end{array}$

